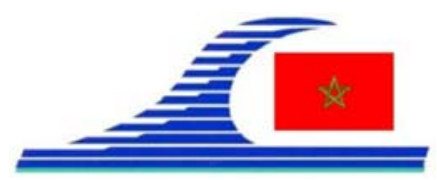

Conférence Méditerranéenne Côtière et Maritime

EDITION 2, TANGER, MAROC (2011)

Coastal and Maritime Mediterranean Conference

Disponible en ligne - http://www.paralia.fr - Available online

\title{
Modélisation numérique de la houle côtière et du transport sédimentaire pour l'étude d'un port de pêche : cas du port de Khemisti
}

\author{
Mustapha Kamel MIHOUBI ${ }^{1,2}$, Hocine DAHMANI ${ }^{2}$, Ahmed KETTAB ${ }^{2}$ \\ Omar KHODJET KESBA ${ }^{1}$
}

\begin{abstract}
1. Laboratoire de Mobilisation et de Valorisation de la Ressource Eau (LMVR-Eau), École Nationale Supérieure d’Hydraulique (ENSH), BP 31, 09000 Blida, Algérie. mihkam@ensh.dz

2. Laboratoire de Recherche des Sciences de l'Eau (LRS-Eau), École Nationale

Supérieure Polytechnique (ENP), avenue Hacène-Badi, BP 182, El Harrach, Algérie.

Kettab@yahoo.fr
\end{abstract}

\section{Résumé:}

Depuis ces dernières années, beaucoup de progrès ont été enregistrés dans le domaine la modélisation numérique des processus côtiers. Il subsiste toutefois quelques difficultés dans les modèles existants pour relier d'une part, l'hydrodynamique de la houle et des courants et d'autre part, le processus de transport sédimentaire et en raison de la variabilité en temps, espace et de l'interaction houle-courant près du rivage.

La présente étude consiste à établir des prévisions du transit sédimentaire à partir de scénarios d'aménagement d'un ouvrage portuaire sur la base d'une modélisation numérique par un modèle hydro-sédimentaire. Dans ce contexte, une étude en mode bidimensionnel d'un aménagement a été établie à l'aide des codes de calcul d'un progiciel de modélisation.

\section{Mots-clés :}

Houle - Courant - Contrainte de radiation - Profil - MIKE21 - Maillage - Sédiment

\section{Introduction}

Dans la zone littorale, des quantités importantes de sable sont transportées sous l'action de la houle et des courants. Quand les vagues atteignent la côte et déferlent sur la plage, elles génèrent des courants côtiers qui interagissent avec les vagues incidentes pour transporter les sédiments (MASSELINK et al., 2006). La houle est un facteur essentiel des mouvements sédimentaires dans la zone du littoral, lors du déferlement, l'énergie des vagues est dissipée et transformée principalement en turbulence qui remet en mouvement les sédiments qui sont transportés par les courants qu'elle engendre et/ou ceux dus aux marées et aux vents. Les interactions entre sédiments et d'eau activent en zone swash, et représente un lieu d'échange et de transport de sédiments (BUTT et al., 2001 ; MIHOUBI, 2008). A ce propos, il est primordial de prévoir l'évolution du transit sédimentaire notamment de la détection des zones d'érosion et de dépôt au niveau du 
La connaissance de la Mer :

un vecteur du développement durable en Méditerranée

rivage. Il est bien évident, que certaines modifications des zones côtières sont liées à l'action anthropique, telles que la réalisation des aménagements hydrotechniques sans tenir compte du régime marin et des interactions avec les différents facteurs énumérés précédemment.

La présente étude consiste à établir des prévisions du transit sédimentaire à partir de scénarios d'aménagement d'un ouvrage portuaire sur la base d'une modélisation numérique avec un modèle hydro-sédimentaire en mode bidimensionnel. Ce modèle a été construit moyennant les codes de calculs MIKE 21 de DHI-Software.

\section{Description de la modélisation}

\subsection{Modèle d'agitation des vagues}

Les modèles de prédiction des vagues sont généralement basés sur l'équation de l'énergie où de la densité de l'énergie de la vague $\mathrm{E}(\sigma, \theta)$, fonction de la pulsation $\omega$ et de la direction $\theta$ de la vague qui varie lentement en fonction de l'espace $(x, y)$ et du temps. En présence d'un courant ambiant, il est plus pertinent de simuler la quantité du mouvement de la vague $\mathrm{N}(\sigma, \theta)$ plutôt l'énergie de la vague:

$$
N(\omega, \theta, x, y, t)=\frac{E(\omega, \theta, x, y, t)}{\sigma}
$$

Le comportement de la houle à l'approche des côtes est modélisé par l'équation de (BERKHOFF, 1972), qui traduit les équations du mouvement en théorie linéaire pour un fond graduellement varié. Elle s'écrit :

$\nabla .\left(C C_{g} \nabla \phi\right)+\frac{C_{g}}{C} \omega^{2} \phi=0$

avec : $\omega^{2}=g k t h(k d)$, relation de dispersion

L'équation (2) peut être résolue directement, par la méthode des éléments finis (MARCER et al., 1994). Ce type de modèle est notamment destiné à étudier les problèmes d'agitation portuaire où les effets de diffraction et réflexion de la houle peuvent être importants.

\subsection{Modèle de transport sédimentaire}

Le modèle proposé repose sur la formulation du Sediment-Transport-Program basée sur une approche de calcul bidimensionnelle horizontale (2DH) dans laquelle, les taux de transport de sédiments sont calculés dans la direction du courant moyen avec une composante transversale résultante de la pente du fond. Les profils verticaux moyens sont calculés en intégrant la contrainte de frottement turbulent:

$\bar{\tau}(z)=\rho v_{t}\left|\frac{\partial \bar{U}}{\partial z}\right|$ 
Le taux total de transport de sédiments non cohésifs $q_{t}$ est calculé en ajoutant les taux de transport de sédiments en suspension $q_{s}$ et en charriage de fond $q_{b}$.

$\left\langle q_{t}\right\rangle=\left\langle q_{s}\right\rangle+\left\langle q_{b}\right\rangle$

\section{Aménagement du port de pêche de Khemisti}

L'abri de pêche de Khemisti est situé à $30 \mathrm{~km}$ de la wilaya de Tipaza, il a connu ces dernières années une grande activité dans le secteur de la pêche. Afin de répondre aux préoccupations de la région une étude d'extension de labri de pêche de Khemisti en port de pêche a été lancée. L’aménagement est protégé par deux petites jetées, la principale située au nord-est $(195 \mathrm{~m})$ et la secondaire à l'ouest $(120 \mathrm{~m})$ la passe d'entrée étant orientée vers le l'ouest. Le code de calcul MIKE 21, est particulièrement utilisé dans l'étude de projets d'aménagement portuaire.

L'analyse de la carte bathymétrique révèle une morphologie sous marine irrégulière entre le trait de côte et l'isobathe $-4 \mathrm{~m}$. Au delà de cette profondeur, la morphologie du fond a tendance à être régulière. Les altitudes min, moyenne, max sont respectivement : $0,00 \mathrm{~m},-22,50 \mathrm{~m},-45,00 \mathrm{~m}$ (figure 1a). La propagation de la houle se traduit à l'approche du rivage par une modification de ces caractéristiques à savoir une diminution de la hauteur de la houle et une déviation de l'angle d'incidence due au phénomène de réfraction (figure $1 \mathrm{~b}$ ). Plusieurs simulations de direction de houles ont été faites.
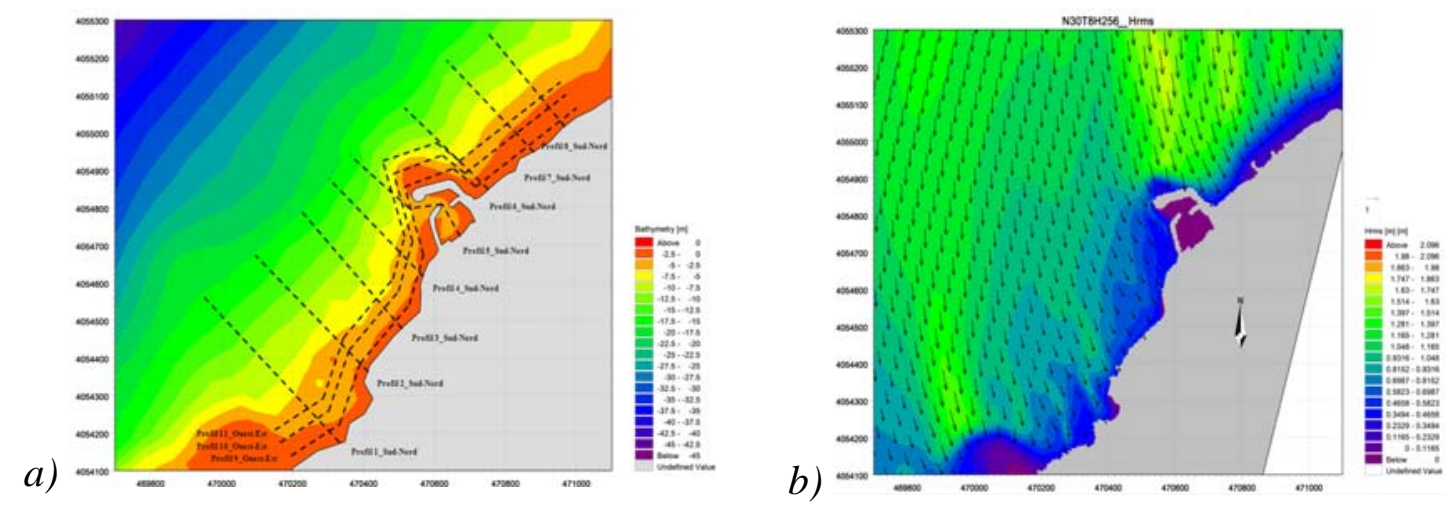

Figure 1 a) Levé bathymétrique de l'aménagement ; b) Epure de réfraction de la houle $\left(M W D=30^{\circ} ; H_{S}=2,56 m ; T=8 s\right)$.

\section{Résultats et discussion}

Après avoir examiné l'état initial du site et mis en évidence les facteurs hydrodynamiques à l'origine de l'ensablement du port, il est clair que les courants littoraux constituent la force motrice du transit sédimentaire suivant le sens dominant des houles incidentes dans la direction ouest-est par rapport à la passe d'entrée. Le dispositif de protection recherché est de permettre de minimiser l'ensablement du port et 
La connaissance de la Mer :

un vecteur du développement durable en Méditerranée

ce à travers diverses variantes d'études d'aménagement. Nous avons ainsi opté après simulation pour une variante à faibles contraintes de radiation à partir de combinaison du modèle hydrodynamique et transport sédimentaire. La solution optimale correspond à un aménagement composé d'un ouvrage épi de $180 \mathrm{~m}$ de longueur et $12 \mathrm{~m}$ de largeur, situé à l'est du port limitant ainsi le flux sédimentaire de même direction (figure 2) protégeant ainsi le bassin du port.

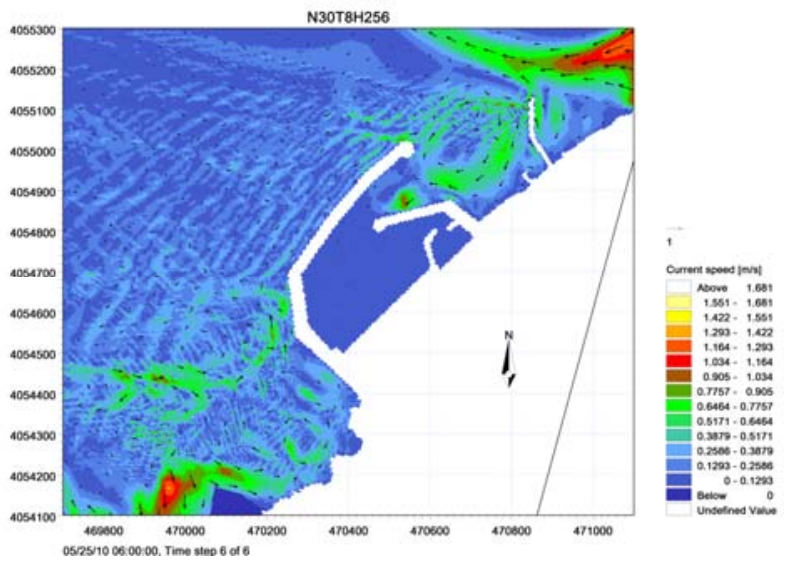

Figure 2. Variante d'aménagement avec un épi sur la coté est du port : conditions de houle: $30^{\circ} \mathrm{N} ; \mathrm{H}_{\mathrm{S}}=2,56 \mathrm{~m} ; \mathrm{T}=8 \mathrm{~s}$.

\section{Remerciements}

Les auteurs remercient vivement, Ahmed BOUDOUMA, Directeur du Laboratoire des Etudes Maritime (LEM) d'Alger pour l'entière collaboration et soutien à la réalisation de ce travail.

\section{Références bibliographiques}

BERKHOFF J.C.W. (1972). Computation of combined refraction-diffraction, Proc. $13^{\text {th }}$ Coastal Engineering Conf., Vancouver, pp 471-490.

BUTT T RUSSEL P.E., TURNER I. (2001). The influence of swash infiltrationexfiltration on beach sediment transport: onshore or offshore? Coastal Engineering, 42 (1), pp 35-52. doi:10.1016/S0378-3839(00)00046-6

MARCER R., LANDEL E., GUERIN P. (1994). Modélisation de l'influence des atténuateurs de houle sur la protection du littoral, $3^{\text {èmes }}$ Journées Nationales Génie Civil-Génie Côtier, Sète, 2-4 Mars, pp 33-37. doi:10.5150/jngcgc.1994.004-M

MASSELINK G., KROON A., DAVIDSON-ARNOTT R.G.D. (2006). Morphodynamics of intertidal bars in wave dominated coastal settings - A review. Geomorphology, 73, 1-2, pp 33-49.

MIHOUBI M.K. (2008). Etude expérimentale de l'interaction eau-sédiment en zone de swash par velocimétrie Doppler ultrasonore(VDU). Thèse de doctorat d'état, Ecole Nationale Polytechnique (ENP), Alger, 210 p. 